\title{
RRM1 predicts clinical outcome of high- and intermediate-risk non-muscle-invasive bladder cancer patients treated with intravesical gemcitabine monotherapy
}

\author{
Zhenxing Yang ${ }^{1}$, Bingqiang Fu², Luqiang Zhou ${ }^{1}$, Jie Xu' ${ }^{1}$, Ping $\mathrm{HaO}^{3}$ and Zhenqiang Fang ${ }^{1 *}$
}

\begin{abstract}
Background: The expression level of ribonucleotide reductase subunit M1 (RRM1) is closely related to the effect of gemcitabine-based therapy in advanced bladder cancer. However, the value of RRM1 expression in predicting progression-free survival in non-muscle-invasive bladder cancer (NMIBC) patients treated with intravesical gemcitabine chemotherapy has not been elucidated.

Methods: This study randomly assigned 162 patients to either the RRM1-known group or the unknown group. We collected cancer tissues from 81 patients to evaluate the mRNA expression of RRM1 by using liquid chip technology. All patients were diagnosed and then treated with intravesical gemcitabine monotherapy immediately after transurethral resection of the bladder tumour (TURBT).

Results: RRM1 expression was high in 21\% (17/81) of patients. The RRM1 mRNA level was not correlated with sex, age, weight, performance status, or CUA/EAU risk $(p>0.05)$. Progression-free survival (PFS) was significantly longer for patients with low RRM1 expression than for patients with high and unknown RRM1 expression $(p=0.009)$. Additionally, the 1- and 2-year relapse rates also differed according to RRM1 expression level. The 1-year relapse rates for RRM1-low, RRM1-high and RRM1-unknown patients were 0, 17.7 and $6.2 \%(p=0.009)$, while the 2-year relapse rates for these groups were $3.1,29.4$, and $11.1 \%(p=0.005)$, respectively.

Conclusions: This preliminary study showed that low RRM1 expression was associated with longer progression-free survival and lower 1-year/2-year relapse rates in NMIBC patients treated with intravesical gemcitabine monotherapy, despite the need for further verification with large sample sizes and considering more mixed factors and biases.
\end{abstract}

Keywords: RRM1, Non-muscle-invasive bladder cancer, Gemcitabine

\section{Background}

Bladder tumours represent the ninth most prevalent malignancy in China, and they were responsible for an estimated 32,900 deaths in 2015 [1]. Approximately 70\% of all bladder carcinomas are first diagnosed as non-muscle-invasive bladder cancer (NMIBC), including tumours of any grade at stages pTa, pT1, or carcinoma in situ (CIS) [2]. Unlike its muscle-invasive counterpart, NMIBC typically has a good prognosis. The EAU guidelines define NMIBC patients as

\footnotetext{
*Correspondence: fangzhenqiang123@126.com

${ }^{1}$ Department of Urology, Second Affiliated Hospital, Third Military Medical University, Chongqing 400037, China

Full list of author information is available at the end of the article
}

low, intermediate, or high risk for recurrence (based upon stage, grade, tumour size, and multifocality) [3]. Patients with higher progression scores are more likely to progress to muscle invasion within 5 years. Transurethral resection of the bladder tumour (TURBT) is the diagnostic and gold standard treatment option for NMIBC. Despite visually complete resection, $30-80 \%$ of NMIBC patients will have disease recurrence, possibly due to invisible residual lesions or implantation of tumour cells during TURBT [4]. Current evidence suggests that subsequent instillations of intravesical chemotherapy are necessary for higher-risk disease [5]. Gemcitabine is a cell-cycle specific antimetabolite that is widely used in intravesical chemotherapy [6]. 
Ribonucleotide reductase subunit M1 (RRM1) is the largest catalytic subunit of ribonucleotide reductase (RR), which is the key enzyme catalysing the transformation of ribonucleoside diphosphates to deoxyribonucleoside diphosphates [7]. Gemcitabine is an analogue of deoxycytidine. The active forms of gemcitabine inhibit DNA synthesis by incorporating into the DNA chain or inhibiting RRM1 activity [8]. Gemcitabine has been widely used for the treatment of several aggressive solid tumour types, including non-small-cell lung cancer (NSCLC), bladder tumours, pancreatic tumours and nasopharyngeal carcinoma [9-12]. There are preclinical and clinical data indicating that high RRM1 protein levels in various cancers are associated with gemcitabine resistance [13, 14]. Moreover, several clinical studies have demonstrated the association between elevated RRM1 levels and unfavourable clinical outcomes in advanced bladder tumour patients treated with gemcitabinebased therapy [9, 15]. However, the relationship between RRM1 mRNA level and gemcitabine activity in NMIBC has not been addressed. In the current paper, we demonstrated the predictive and prognostic value of RRM1 in patients with NMIBC receiving intravesical gemcitabine chemotherapy.

\section{Methods}

\section{Patients}

This retrospective study enrolled 162 patients with histological confirmed NMIBC and intermediate/highrisk disease at the Second Affiliated Hospital of Third Military Medical University from November 2010 to January 2016. Tissue samples from patients were obtained after surgery. An Eastern Cooperative Oncology Group (ECOG) performance status (PS) of 0 to 2 was assessed in all enrolled patients. Patient inclusion criteria included the following: 1) an NMIBC patient diagnosis following the EAU guidelines; 2) intermediate or highrisk bladder cancer patients without lymph node metastasis or distant metastases; 3) all patients underwent transurethral resection of the bladder tumour plus subsequent instillations of intravesical gemcitabine chemotherapy; 4) first diagnosis of a bladder tumour without accepting any surgery or drug treatment; and 5) patients voluntarily participated in the study and signed the informed consent form. This study was conducted with the approval of the medical ethics committee of Second Affiliated Hospital of the Third Military Medical University. Each patient provided written informed consent before participation in the current investigation. Patient information on pathologic characteristics, treatment details, and survival was obtained from follow-up and surgical records. The major clinical endpoint in the current study was disease-free survival (DFS), and the secondary clinical outcomes were 1-year and 2-year relapse rates.

\section{Treatment and response evaluation}

All patients received intravesical gemcitabine monotherapy immediately after TURBT. A total of $1000 \mathrm{mg}$ of gemcitabine was diluted in $40 \mathrm{ml}$ of saline solution, and patients received weekly instillations for 8 consecutive weeks. The drug was held in the bladder for $60 \mathrm{~min}$. The treatment cycle was then changed to once a month for one year. Follow-up was performed to assess the efficacy of the treatment for all involved patients. In general, in the first year, cystoscopy and urinary cytology were examined at 3-month intervals and then at 6-month intervals in the next year. Relapse was defined as a positive examination on cystoscopy. The first recurrence (disease-free survival) time was defined as the period between TURBT and positive finding during cystoscopy.

\section{RRM1 mRNA expression analysis}

In the current investigation, multiplex branched-DNA (bDNA) liquid chip technology was employed to perform RRM1 expression analyses at SurExam Medical Test Centre, Guangzhou, China, and these analyses are detailed in the paper published by Zhang [16]. Briefly, digested tissue was incubated with target gene-specific probe sets, and then fluorescence capture beads were added. Then, the mixture was hybridized with bDNA signal amplification probes for the purpose of signal amplification. In the end, the Luminex 200 system (Luminex Corp., Austin, Texas) was used to cluster the fluorescence value of each sample. Three standard genes were demonstrated as reference genes, including beta-2microglobulin (B2M), transfer in receptor (TFRC), and TATA box-binding protein (TBP). All original data were subjected to standardize processing, which included raw data alignment (fastq file), duplication removal, quality control, etc., until we obtained clean data for subsequent analyses. All gene expression levels among patients were distributed across the whole samples, and then each patient received an RRM1 expression value. The median RRM1expression level was selected for the cutoff value. The expression level of RRM1 was considered high if its level was equal to or exceeded the cutoff value. All other mRNA values were considered low expression.

\section{Statistical analysis}

SPSS statistical software, version 19.0 (IBM Corporation, Armonk, New York, USA) was employed for data analysis. The $X^{2}$ and Fisher exact tests were performed to identify associations between clinicopathologic variables and RRM1 status as appropriate. Survival curves were demonstrated by using the Kaplan-Meier method. A logrank test was used to compare survival differences among groups. All statistical tests were two-sided, and a statistically significant difference was defined as $p<0.05$. 


\section{Results}

\section{Patient features}

Table 1 shows the baseline clinicopathologic features of the study population. The median age of the 162 patients was 60 years (range, 25-89 years), and there were $132(81.5 \%)$ men and $30(18.5 \%)$ women in the cohort. All patients were classified as having high-risk NMIBC and an ECOG PS $0 \sim 2$. All patients underwent 1 year of intravesical gemcitabine chemotherapy after TURBT. The median patient follow-up was 30.5 months (range, 14-76 months). The patients were randomly assigned evenly to either the RRM1-known group (high and low expression of RRM1) or to the unknown group. There were 81 tumour samples successfully processed for RRM1 analysis, and 21.0\% (17/81) of the patient demonstrated high RRM1 expression.

\section{Relationship between clinicopathologic features and RRM1 expression}

The expression of RRM1 was divided into high and low expression according to the cutoff value of RRM1, which was 0.557 (range 0.006-0.997). Of the 162 patients, RRM1 expression was high in 17 (10.5\%) patients and low in $64(39.5 \%)$ patients. The RRM1 level was unknown in the remaining $50 \%$ of patients. As shown in Table 2, the level of RRM1 expression was moderately associated with clinicopathologic features. There were no significant differences observed in sex $(p=0.921)$, age

Table 1 Patients' characteristics according to RRM1 expression

\begin{tabular}{|c|c|c|c|c|c|c|}
\hline \multirow[t]{2}{*}{ Features } & \multirow[t]{2}{*}{$N$} & \multicolumn{3}{|c|}{ RRM1 expression } & \multirow[t]{2}{*}{$\lambda^{2}$ 值 } & \multirow[t]{2}{*}{$p$} \\
\hline & & Low (\%) & High (\%) & Unknown (\%) & & \\
\hline \multicolumn{7}{|l|}{ Gender } \\
\hline Female & 30 & $11(17.2)$ & $3(17.6)$ & $16(19.8)$ & 0.166 & 0.921 \\
\hline Male & 132 & $53(82.8)$ & $14(82.4)$ & $65(80.2)$ & & \\
\hline \multicolumn{7}{|l|}{ Age (years) } \\
\hline$\leq 60$ & 85 & $39(60.2)$ & $12(70.6)$ & $34(42.0)$ & 3.542 & 0.170 \\
\hline$>60$ & 77 & $25(39.8)$ & $5(29.4)$ & $47(58.0)$ & & \\
\hline \multicolumn{7}{|c|}{ Weight (Kg) } \\
\hline$\leq 65$ & 78 & $26(40.6)$ & $8(47.1)$ & $44(56.4)$ & 2.695 & 0.260 \\
\hline$>65$ & 84 & $38(59.4)$ & $9(52.9)$ & $37(44.0)$ & & \\
\hline \multicolumn{7}{|c|}{ ECOG Score } \\
\hline $0-1$ & 157 & $62(96.9)$ & $17(100.0)$ & $78(96.3)$ & 0.645 & 0.724 \\
\hline 2 & 5 & $2(3.1)$ & 0 & $3(3.7)$ & & \\
\hline \multicolumn{7}{|c|}{ CUA/EAU risk group } \\
\hline 2 & 54 & $22(34.4)$ & $6(35.3)$ & $26(32.1)$ & 0.116 & 0.944 \\
\hline 3 & 108 & $42(65.6)$ & $11(64.7)$ & $55(67.9)$ & & \\
\hline \multicolumn{7}{|l|}{ Relapse } \\
\hline Yes & 23 & $3(4.9)$ & $5(29.4)$ & $15(18.5)$ & 9.223 & 0.010 \\
\hline No & 139 & $61(95.1)$ & $12(70.6)$ & $66(81.5)$ & & \\
\hline
\end{tabular}

$p<0.05$ is set in boldface
Table 2 The 1-year/2-year relapse rates according to RRM1 expression and clinicopathological features

\begin{tabular}{|c|c|c|c|c|c|}
\hline & No & 1-y R $\mathrm{R}^{\mathrm{a}}$ rate $(\%)$ & $p$ & 2-y $R^{a}$ rate $(\%)$ & $p$ \\
\hline \multicolumn{6}{|l|}{ RRM1 level } \\
\hline Low & 64 & 0 & 0.009 & $3.1 \%$ & 0.005 \\
\hline High & 17 & $17.7 \%$ & & $29.4 \%$ & \\
\hline Unknown & 81 & $6.2 \%$ & & $11.1 \%$ & \\
\hline \multicolumn{6}{|l|}{ Gender } \\
\hline Female & 30 & $13.3 \%$ & 0.040 & $13.3 \%$ & 0.500 \\
\hline Male & 132 & $3.0 \%$ & & $9.1 \%$ & \\
\hline \multicolumn{6}{|l|}{ Age (years) } \\
\hline$\leq 60$ & 85 & $4.7 \%$ & 0.569 & $10.6 \%$ & 0.75 \\
\hline$>60$ & 77 & $5.3 \%$ & & $9.1 \%$ & \\
\hline \multicolumn{6}{|l|}{ Weight (Kg) } \\
\hline$\leq 65$ & 78 & $13.3 \%$ & 0.204 & $7.7 \%$ & 0.369 \\
\hline$>65$ & 84 & $4.8 \%$ & & $11.9 \%$ & \\
\hline \multicolumn{6}{|l|}{ ECOG Score } \\
\hline $0-1$ & 157 & $5.1 \%$ & 0.774 & $10.2 \%$ & 0.452 \\
\hline 2 & 5 & 0 & & 0 & \\
\hline \multicolumn{6}{|c|}{ CUA/EAU risk group } \\
\hline 2 & 54 & $1.9 \%$ & 0.271 & $11.1 \%$ & 0.710 \\
\hline 3 & 108 & $6.5 \%$ & & $9.3 \%$ & \\
\hline
\end{tabular}

( $p=0.170)$, weight $(p=0.260)$, ECOG performance status ( $p=0.610)$, or CUA/EAU risk between the three groups $(p=0.944)$. Surprisingly, when compared to patients with low $(4.9 \%, 3 / 64)$ and unknown $(18.5 \%, 15 / 81)$ RRM1 levels, patients with high RRM1 levels were more likely to relapse $(29.4 \%, 5 / 17), p=0.010$.

\section{Association between RRM1 level and clinical outcomes}

After a median follow-up of 30.5 months, 14.2\% (23/162) of the patients experienced disease progression, and all patients were alive. As shown in Fig. 1, PFS was significantly longer for patients with low RRM1 expression than for patients with high and unknown RRM1 expression, $p=0.009$. However, median PFS could not be calculated because a majority of patients were disease-free at the end of follow-up.

Additionally, the 1-year and 2-year relapse rates also differed according to RRM1 expression and other clinical characteristics. The 1-year relapse rates for RRM1-low, RRM1high and RRM1-unknown patients were 0, 17.7 and 6.2\% $(p=0.009)$, respectively, while the 2 -year relapse rates in these groups were $3.1,29.4$, and $11.1 \%(p=0.005)$, respectively. Other clinical characteristics, such as age, weight, and performance status, showed little association with 1-year/2year relapse rates. The female patients had higher 1-year relapse rates than the male patients $(13.3 \%$ vs. $3.0 \%, p=0.04)$. 


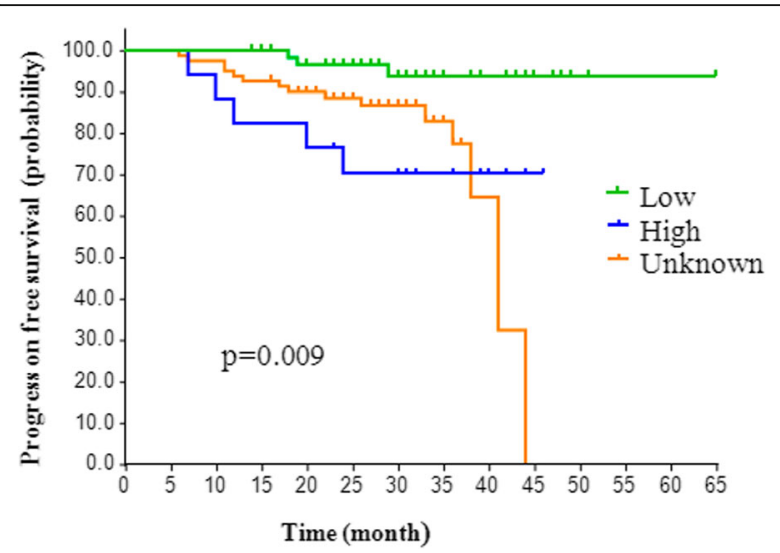

Fig. 1 Analysis of the progression of disease-free survival probability among low, high and unknown groups

\section{Discussion}

In clinical practice, the effects of chemotherapeutic agents or regimens vary among different individuals. Pharmacogenomics studies have shown that genetic factors play a vital role in curative effects. Therefore, identifying a biomarker is essential for establishing personalized treatments and improving treatment outcomes. Several recent studies have focused on evaluating predictive and/or prognostic markers for various tumours, including non-small-cell lung cancer (NSCLC), nasopharyngeal carcinoma and bladder carcinoma.

Our present study investigated the predictive and prognostic value of RRM1 in patients with NMIBC receiving intravesical gemcitabine chemotherapy. There were 162 NMIBC patients randomly assigned to either the RRM1-known group or the unknown group. The expression levels of RRM1 were significantly associated with age, sex, weight, ECOG performance status, or CUA/EAU risk, which is in accordance with prior results in advanced UC and NSCLC $[9,17]$. Our data showed that high RRM1 expression was observed in less than $30 \%$ of tumours and was an unfavourable prognostic factor for PFS. Conversely, a low RRM1 level was found to be correlated with better PFS according to the KaplanMeier analysis. In addition, patients with low RRM1 expression showed the lowest 1-year/2-year relapse rate, while the RRM1-high patients had the highest relapse rates (17.1 and 29.4\%, respectively). The association between RRM1 expression and prognosis in the present study was consistent with previous studies investigating other tumours treated with gemcitabine.

Previous studies associated with RRM1 expression in NSCLC patients largely demonstrated that low or negative RRM1 levels in patients with advanced NSCLC receiving gemcitabine-based regimens were correlated with higher response rates and a better prognosis [18]. In addition, there are also several studies that evaluated the predictive and/or prognostic value of RRM1 expression level in patients with urothelial carcinoma (UC). In one study, high RRM1 expression in respectable MIBC patients aged $<70$ years was associated with improved survival [19]. On the other hand, the RRM1 level in advanced $\mathrm{BC}$ patients receiving gemcitabine-based regimes was not correlated with response or OS, except time-toprogression (TTP) [20]. These discrepant results may be attributable to differences in the patients involved. Similar to our results, Kim et al. found that high RRM1 expression was associated with inferior prognosis and clinical outcome after platinum plus gemcitabine combination chemotherapy for advanced UC $[9,15]$. In contrast with previous studies regarding RRM1 in UC with gemcitabine-based therapy, this is the first study of early $\mathrm{BC}$ (NMIBC) with intravesical gemcitabine monotherapy. Taken together, these studies suggest that low RRM1 expression may help identify patients who will significantly benefit from gemcitabine-based chemotherapy in early and advanced UC.

The role of RRM1 in gemcitabine resistance carries more significance in the MIBC population, in which gemcitabine chemotherapy in combination with cisplatin is the standard of care. The standard treatment of NMIBC, however, remains treatment with bacillus Calmette-Guerin (BCG). The role of gemcitabine as a standard intravesical treatment in non-BCG refractory patients is unclear, as recurrence rates have been shown to be significantly higher among high-risk patients treated with gemcitabine compared with those treated with BCG. BCG was the first choice in intravesical instillation treatment with intermediate- or high-risk NMIBC. The recurrence rate of BCG treatment is lower than that of gemcitabine intravesical chemotherapy. However, in China, the price of BCG is too high, approximately 6 times higher than the cost of gemcitabine treatment, and no medical insurance covers this cost. For economic reasons, most NMIBC patients generally give up using BCG treatment and use gemcitabine intravesical chemotherapy as a secondary choice. The purpose of current study was to maximize the efficacy of gemcitabine perfusion therapy in these patients.

Nevertheless, limitations must be considered in the current investigation. The current study is a singlecentre study, and the number of RRM1-high patients investigated was relatively small $(N=17)$. In addition, the Kaplan-Meier analysis of RRM1-low and RRM1unknown groups failed to reach median PFS despite having a median follow-up of 30.5 months (range, 14-76 months). One main reason for these limitations is that the prognosis of NMIBC patients is usually good. Therefore, further well-designed studies must enrol a larger sample size and have longer follow-up periods. In addition, the nature of the retrospective study means 
that there is a risk of selection bias, which is another limitation in our investigation, and a lack of secondary analysis (regression models) to confirm RRM1 as an independent variable associated with disease recurrence and progression should be avoided in future studies. Despite these limitations, the current study is clinically meaningful and suggests the important role of RRM1 mRNA expression in patients with NMIBC. To our knowledge, this is the first study evaluating RRM1 mRNA in NMIBC patients treated with intravesical gemcitabine monotherapy.

\section{Conclusions}

In conclusion, this preliminary study showed that low RRM1 expression was associated with longer progression-free survival and lower 1-year/2-year relapse rates in NMIBC patients treated with intravesical gemcitabine monotherapy, despite the need for further verification with large sample sizes and considering more mixed factors and biases.

\section{Abbreviations}

CUA: China Urological Association; EAU: European Association of Urology; NMIBC: Non-muscle-invasive bladder cancer; PFS: Progression-free survival; RRM1: Ribonucleotide reductase subunit M1; TURBT: Transurethral resection of the bladder tumour

\section{Acknowledgements}

Not applicable.

\section{Authors' contributions}

Z.F., J.X., and P.H. designed the investigation; Z.F. and J.X. provided the funding for the current research; Z.F. and L.Z. had access to all tissues samples; Z.F. and Z.Y.J.X. obtained statutory and ethics approvals; B.F. and Z.Y. contributed to data acquisition; Z.Y., B.F., and Z.F. performed the data preparation, quality control and analyses and checked the results. Y. and B.F. drafted the report. All authors contributed to writing the final report and approved the version to be published.

\section{Funding}

The current work was supported partly by the clinical research project key projects of Second Affiliated Hospital, Third Military Medical University (2014YLC28 for purchasing most of the experimental reagents, materials and sequencing fees) and partly by the National Natural Science Foundation of China (81772702 for providing the labour costs for the researchers and the fee for some antibodies).

\section{Availability of data and materials}

The datasets for the current study are available from the corresponding author upon reasonable request.

\section{Ethics approval and consent to participate}

This study was conducted with the approval of the medical ethics committee of Second Affiliated Hospital of the Third Military Medical University. Each patient signed a written informed consent form before entry into this study.

\section{Consent for publication}

Not applicable.

\section{Competing interests}

The authors declare that they have no competing interests.

\section{Author details}

${ }^{1}$ Department of Urology, Second Affiliated Hospital, Third Military Medical University, Chongqing 400037, China. ${ }^{2}$ SurExam Bio-Tech Co, Guangzhou 510663, Guangdong, China. ${ }^{3}$ Department of Oncology, Second Affiliated Hospital, Third Military Medical University, Chongqing 400037, China.

Received: 15 January 2019 Accepted: 8 July 2019

Published online: 24 July 2019

\section{References}

1. Chen W, Zheng R, Baade PD, Zhang S, Zeng H, Bray F, Jemal A, Yu XQ, He J. Cancer statistics in China, 2015. CA Cancer J Clin. 2016;66(2):115-32.

2. Traboulsi SL, Kassouf W. Bladder cancer: a step closer to individualized treatment for bladder cancer. Nat Rev Urol. 2016;13(3):127-8.

3. Olivier Bosset $P$, Neuzillet $Y$, Paoletti $X$, Molinie $V$, Botto $H$, Lebret $T$. Longterm follow-up of TaG1 non-muscle-invasive bladder cancer. Urol Oncol. 2015;33(1):20 e21-7.

4. Porten SP, Leapman MS, Greene KL. Intravesical chemotherapy in nonmuscle-invasive bladder cancer. Indian J Urol. 2015;31(4):297-303.

5. Sylvester RJ, Oosterlinck W, van der Meijden AP. A single immediate postoperative instillation of chemotherapy decreases the risk of recurrence in patients with stage ta T1 bladder cancer: a meta-analysis of published results of randomized clinical trials. J Urol. 2004;171(6 Pt 1):2186-90 quiz 2435.

6. Kim SI, Choo SH. Intravesical Chemotherapy. In: Bladder Cancer.edn: Elsevier; 2018. p. 263-76. https://doi.org/10.1016/B978-0-12-809939-1.00019-9.

7. Brissenden J, Caras I, Thelander L, Francke U. The structural gene for the M1 subunit of ribonucleotide reductase maps to chromosome 11, band p15, in human and to chromosome 7 in mouse. Exp Cell Res. 1988;174(1):302-8.

8. Mini E, Nobili S, Caciagli B, Landini I, Mazzei T. Cellular pharmacology of gemcitabine. Ann Oncol. 2006;17(Suppl5):v7-12.

9. Kim M, Ku JH, Kwak C, Kim HH, Lee E, Keam B, Kim TM, Heo DS, Lee SH, Moon KC. Predictive and prognostic value of ribonucleotide reductase regulatory subunit $\mathrm{M} 1$ and excision repair cross-complementation group 1 in advanced urothelial carcinoma (UC) treated with first-line gemcitabine plus platinum combination chemotherapy. PLoS One. 2015;10(7):e0133371.

10. Zhao LP, Xue C, Zhang JW, Hu ZH, Zhao YY, Zhang J, Huang Y, Zhao HY, Zhang L. Expression of RRM1 and its association with resistancy to gemcitabine-based chemotherapy in advanced nasopharyngeal carcinoma. Chinese journal of cancer. 2012;31(10):476-83.

11. Lee JJ, Maeng CH, Baek SK, Kim GY, Yoo JH, Choi CW, Kim YH, Kwak YT, Kim $\mathrm{DH}$, Lee $\mathrm{YK}$ et al: The immunohistochemical overexpression of ribonucleotide reductase regulatory subunit M1 (RRM1) protein is a predictor of shorter survival to gemcitabine-based chemotherapy in advanced non-small cell lung cancer (NSCLC). Lung cancer (Amsterdam, Netherlands) 2010, 70(2):205-210.

12. Aoyama T, Miyagi Y, Murakawa M, Yamaoku K, Atsumi Y, Shiozawa M, Ueno M, Morimoto M, Oshima T, Yukawa N, et al. Clinical implications of ribonucleotide reductase subunit $\mathrm{M} 1$ in patients with pancreatic cancer who undergo curative resection followed by adjuvant chemotherapy with gemcitabine. Oncol Lett. 2017;13(5):3423-30.

13. Davidson JD, Ma L, Flagella M, Geeganage S, Gelbert LM, Slapak CA. an increase in the expression of ribonucleotide reductase large subunit 1 is associated with gemcitabine resistance in non-small cell lung cancer cell lines. Cancer Res. 2004;64(11):3761-6.

14. Bergman AM, Eijk PP, Ruiz van Haperen WW, Smid K, Veerman G, Hubeek I van den ljssel P, Ylstra B, Peters GJ. in vivo induction of resistance to gemcitabine results in increased expression of ribonucleotide reductase subunit M1 as the major determinant. Cancer Res. 2005:65(20):9510-6.

15. Shilkrut M, Wu A, Thomas DG, Hamstra DA. Expression of ribonucleoside reductase subunit $\mathrm{M1}$, but not excision repair cross-complementation group 1 , is predictive in muscle-invasive bladder cancer treated with chemotherapy and radiation. Mol Clin Oncol. 2014;2(3):479-87.

16. Zhang Q, Sun T, Kang P, Qian K, Deng B, Zhou J, Wang R, Jiang B, Li K, Liu $F$, et al. Combined analysis of rearrangement of ALK, ROS1, somatic mutation of EGFR, KRAS, BRAF, PIK3CA, and mRNA expression of ERCC1, TYMS, RRM1, TUBB3, EGFR in patients with nonsmall cell lung cancer and their clinical significance. Cancer Chemother Pharmacol. 2016;77(3):583-93.

17. Simon G, Sharma A, Li X, Hazelton T, Walsh F, Williams C, Chiappori A, Haura E, Tanvetyanon T, Antonia S, et al. Feasibility and efficacy of molecular analysis-directed individualized therapy in advanced non-small-cell lung 
cancer. Journal of clinical oncology : official journal of the American Society of Clinical Oncology. 2007;25(19):2741-6.

18. Gong W, Zhang X, Wu J, Chen L, Li L, Sun J, Lv Y, Wei X, Du Y, Jin H et al: RRM1 expression and clinical outcome of gemcitabine-containing chemotherapy for advanced non-small-cell lung cancer: a meta-analysis. Lung cancer (Amsterdam, Netherlands) 2012, 75(3):374-380.

19. Harshman LC, Bepler G, Zheng Z, Higgins JP, Allen Gl, Srinivas S. Ribonucleotide reductase subunit M1 expression in resectable, muscleinvasive urothelial cancer correlates with survival in younger patients. BJU Int. 2010;106(11):1805-11.

20. Bellmunt J, Paz-Ares L, Cuello M, Cecere FL, Albiol S, Guillem V, Gallardo E, Carles J, Mendez P, de la Cruz JJ, et al. Gene expression of ERCC1 as a novel prognostic marker in advanced bladder cancer patients receiving cisplatinbased chemotherapy. Ann Oncol. 2007;18(3):522-8.

\section{Publisher's Note}

Springer Nature remains neutral with regard to jurisdictional claims in published maps and institutional affiliations.

Ready to submit your research? Choose BMC and benefit from:

- fast, convenient online submission

- thorough peer review by experienced researchers in your field

- rapid publication on acceptance

- support for research data, including large and complex data types

- gold Open Access which fosters wider collaboration and increased citations

- maximum visibility for your research: over $100 \mathrm{M}$ website views per year

At BMC, research is always in progress.

Learn more biomedcentral.com/submissions 